\title{
Objectively and Subjectively Measured Effects of a Palmitoleate Dietary Supplement: A Placebo- Controlled Double-Blind Randomized Crossover Clinical Trial
}

\author{
Masa Sasagawa ( $\nabla$ msasagawa@bastyr.edu ) \\ Bastyr University https://orcid.org/0000-0002-9047-1388 \\ Miranda J Boclair \\ Bastyr University \\ Paul S Amieux \\ Bastyr University
}

\section{Research}

Keywords: palmitoleate, palmitoleic acid, omega-7 fatty acid, n7FA, RCT, dietary supplement, inflammatory biomarkers, subjective evaluation, placebo effect

Posted Date: December 3rd, 2020

DOI: https://doi.org/10.21203/rs.3.rs-118804/v1

License: () (1) This work is licensed under a Creative Commons Attribution 4.0 International License. Read Full License 


\section{Abstract}

Background: Supplementation with dietary palmitoleate was reported to lower serum high-sensitivity Creactive protein (hsCRP) for those with elevated hsCRP. The objective of the study was to evaluate subjectively and objectively measured outcomes of an omega-7 fatty acid (n7FA) dietary supplement on people with non-specific chronic musculoskeletal discomfort.

Methods: A double-blind, placebo-controlled, 1:1 randomized single crossover trial was composed of Group "A" which was given placebo first and verum second while group "B" was given verum first and placebo second. Volunteers with uncomplicated chronic musculoskeletal discomfort or a baseline serum hsCRP $>1.0$ $\mathrm{mg} / \mathrm{L}$ were recruited from 2017 - 2019. Verum capsules contained palmitoleate and the placebo contained medium chain fatty acids (MCT). Participants took two capsules per day from the first bottle for three weeks followed by two capsules per day from the second bottle without washout for an additional three weeks. Subjects were instructed to maintain their current diet and lifestyle.

Results: From 211 volunteers consented and screened, 56 subjects were randomized. Six subjects dropped out, and fifty subjects contributed to the statistical analyses. At baseline, none of the investigated biomarkers were significantly correlated to subjectively assessed musculoskeletal discomfort levels, and Group B showed higher HDL and lower triglyceride than Group A. Main Outcomes of crossover analysis found no statistical significance in all measurements. The mean difference of subjectively measured PROMIS ${ }^{\circledR}$ scores between the first and second visits showed improvement for both placebo and verum groups.

Conclusions: Supplementation for three weeks with $688 \mathrm{mg}$ per day of a palmiteolate dietary supplement did not reduce serum inflammatory biomarkers nor did it improve subjectively measured quality of life (QoL) for those with chronic non-specific musculoskeletal discomfort. Future studies should explore time, dosing and verification of cis-palmiteolate versus trans-palmiteolate. Future studies should also consider selection of patients with chronic inflammation, for example individuals with higher body-mass index (BMI).

Trial registration: NCT03669575. Registered 13 September 2018 - Retrospectively registered, https://clinicaltrials.gov/ct2/show/NCT03669575.

\section{Introduction}

In the U.S., cytotoxic or noble agents must be tested for therapeutic use by well-designed and phased trials as specified by the FDA, which oversees safety, tolerability and efficacy requirements ${ }^{1}$. On the other hand, dietary supplements and other well-known essential nutrients are not required by law to follow the FDA phased trial guidelines and are thus not allowed to advertise any intention to diagnose, treat, cure, prevent or mitigate a clinical condition. Dietary supplements must be advertised the above limitation so that they do not violate FTC regulations. Because of this legal ability to avoid the normal process of clinical evaluation and validation, establishing a solid and compelling evidence-informed or evidence-based medicine (EBM) for dietary supplements and other lifestyle/dietary modalities has been challenging. 
In this article, in order to clarify what is referred to as complementary and integrative health $(\mathrm{ClH})$ versus complementary and alternative medicine (CAM), we propose that CAM focuses on modalities, whereas $\mathrm{CIH}$ refers to a setting or environment that may provide a combination of modalities but also embodies diverse alternative health philosophies and principles. In many cases, CAM trials are conducted with the hope of validating traditional and anecdotal health uses or to validate the health claims of a product. However, scientific rigor in many of these studies has often been lacking for a variety of reasons, including: 1) lack of financial support ${ }^{2}, 2$ ) lack of both research infrastructure and competent researchers at $\mathrm{ClH}$ institutions ${ }^{3}$, and 3) influence exerted by interest groups ${ }^{4}$. Current research best practices often includes outcome measurements that are subjective (e.g., Patient Reported Outcomes) in order to reflect the values and preferences of patients ${ }^{5}$, and the inclusion of both subjective and objective measurements may enhance scientific rigor when moving toward a fully EBM model of research on CAM.

A double-blind, placebo-controlled, 1:1 randomized single crossover (3 weeks $\times 2$ ) trial of an omega-7 fatty acid (n7FA) supplement containing palmitoleate was conducted from 2017-2019. The investigational supplement was provided by Barlean's Organic Oils, LLC (Ferndale, WA), which was also a sponsor of this trial, together with Bastyr University Research Institute. The purpose of the trial was to test the hypothesis that supplementation with a n7FA-containing oil alleviates musculoskeletal discomfort and reduces serum inflammatory markers. The purported active constituent of the verum was palmitoleate, which is a 16-carbon monounsaturated (16:1) fatty acid with a double bond occurring at the 7th carbon atom away from the methyl group. There is no Recommended Daily/Dietary Allowance (RDA) for n7FA, which is not an essential fatty acid, meaning the body can produce it de novo ${ }^{6}$. The aim of this study was to enroll sufficient numbers of eligible volunteers to reach 50 participants who would provide data on three time points. This clinical trial was registered on ClinicalTrials.gov (NCT03669575) and conducted in accordance with the International Council of Harmonisation of Technical Requirements for Pharmaceuticals for Human Use (ICH) and Good Clinical Practice (GCP). The protocol was approved by the Bastyr University Institutional Review Board (IRB\#16-1575).

\section{Materials And Methods}

Administrative considerations

Because the sponsor company is a longtime supporter of the university, the Office of Research Integrity (ORI) required that the research team to maintain a strict and conscientious boundary between the sponsor organization and the research team. Our ORI is the administrative office for the IRB and oversees the scientific and ethical integrity of research, including managing any potential conflict of interest. The a priori protocol and double blinding were strictly maintained, and all acquired data per protoco/ were used for the analysis. All analyses, including tallying adverse events, dropouts and crossover analyses were performed while a statistician was given the A or B designations only. Outliers were detected by the Grubb's Extreme Studentized Deviate (ESD) test. The code was broken in a group meeting after all analyses were completed. The trial took place at the Clinical Research Center at Bastyr University in Kenmore, Washington.

Double blinding and randomization 
The investigational products were transported to the trial site with dosing and storage instructions. Each bottle was stamped with a unique lot number, which distinguished a placebo or verum bottle identifiable by a single designated researcher. All staff including the PI were blinded to the bottle identity. Fifty-six bottles of the verum and 56 bottles of placebo (112 bottles of study product) were prepared. Fourteen blocks of four ( 2 verum and 2 placebo) were randomized as $A$ or $B$, assuring an equal number of participants assigned to both groups for every four subjects enrolled ${ }^{7}$. Each participant received one of the verum and one of the placebo bottles based on the sequence defined by A or B designation.

Verum and placebo

Per the certificate of analysis, the verum capsule contained $413 \mathrm{mg}$ omega-7 Fish Oil Blend (Barlean's) derived from Alaskan Pollock (Gadus Chalcogrammus) consisting of $227 \mathrm{mg}$ palmitoleate per capsule. The rancidity of the oil was measured by the level of peroxide and p-Anisidine, and indicated as $1.16 \mathrm{mEq} / \mathrm{kg}$ and $3.91 \mathrm{mEq} / \mathrm{kg}$, respectively. Peroxide values (PVs) under $10-20 \mathrm{mEq} / \mathrm{kg}$ of fish oil are considered very fresh and unlikely to exhibit rancid smells ${ }^{8}$. Hung and Slinger (1980) have indicated that for salmon oil, PVs around $26 \mathrm{mEq} / \mathrm{kg}$ is 'moderately oxidized,' $120 \mathrm{mEq} / \mathrm{kg}$ is 'highly oxidized,' and $314 \mathrm{mEq} / \mathrm{kg}$ is 'extremely oxidized' $^{\prime}$. Placebo capsules contained the equivalent quantity of fatty acid in the form of medium chain triglycerides (MCT) consisting of $65 \%$ caprylic acid (8:0), $45 \%$ capric acid (10:0), and $<2 \%$ lauric acids (12:0) per the company's information. The investigational products were stored at room temperature in a locked closet.

Outcome measurements

To assure sufficient statistical power for the primary serum biomarkers of inflammation, we modeled the previously studied biomarker used for n7FA, which was high-sensitivity C-reactive protein (hsCRP). Bernstein et al. (2014) used a pill containing "purified" $52.5 \%$ cis-palmitoleate, $220.5 \mathrm{mg}$ from "double metabolically distilling anchovy oil after the removal of omega-3s" and used a dose of one pill per day for 30 days $\mathrm{s}^{10}$. According to the reported intervention effect estimate of $-1.9 \mathrm{mg} / \mathrm{L}$ and a conservative estimate of a standard deviation of $0.2 \mathrm{mg} / \mathrm{L}$ between verum and placebo, only four subjects would be needed to reach $95 \%$ power at the alpha level of $0.05^{11}$. A recruitment size of 56 was chosen for secondary biomarker outcomes, which included Tumor Necrosis Factor alpha (TNFa) and interleukin-6 (IL-6) from the published literature ${ }^{12-14}$. Using a crossover design, 28 participants received the verum first and another group of 28 participants received the placebo first, and this data would then be analyzed by a mixed pre-post t-test. Blood was collected from the antecubital vein. Several factors that deviated from Bernstein's study were: 1) our study did not recruit subjects with a serum hsCRP range of $2-5 \mathrm{mg} / \mathrm{L}, 2)$ the dosage was tripled, 3) the length of study was 21 days, and 4) our study did not confirm the cis-isoform of n7FA. In addition to the objective outcomes, three subjective measurements were selected from the questionnaire-bank of the REDCap database system ${ }^{15}$, which were: 1) fatigue, 2) pain interference, and 3) physical function domains from the Patient-Reported Outcomes Measurement Information System (PROMIS $\left.{ }^{1}\right)^{16}$. These instruments were filled out at the baseline, the first follow-up and the last visit.

Trial design, procedures, and sequence of events 
The trial was characterized by the following: 1) receiving voluntary contact, 2) consenting, 3) the baseline assessment, 4) no run-in re-assessment, 5) taking two gel capsules a day from the first assigned bottle for three weeks, 6) returning for the first follow up assessment, 7) no washout period, 8) taking two gel capsules a day from the second assigned bottle for three weeks, 9) returning for the last assessment.

\section{Crossover design and statistics}

The crossover design aims to eliminate between-subject variability of pre-post as well as verum-placebo effects; however, the level of sensitivity and the power of statistical calculation are determined by three different assumptions between the verum and placebo responses: 1) having a positive correlation, 2) having no correlation, or 3 ) having a negative correlation ${ }^{17}$. A positive correlation between the verum and the placebo means that if you have a large placebo effect, your verum effect will also be greater. A negative correlation between the verum and the placebo means that a high placebo responder will have a smaller effect for the verum. In this study, no correlation was assumed.

\section{Results}

Of the 211 subjects consented and screened, 56 subjects were randomized. Five subjects dropped out during the verum dosing and one during the placebo dosing, which resulted in 50 subjects as shown in Fig. 1. At the baseline, twelve subjects (24\%) were using some sort of dietary supplement such as a multivitamin, fish oil, turmeric; alone or in combination with other pharmaceuticals. Non-steroidal anti-inflammatory drug use was $16 \%$; prescriptions of muscle relaxants or non-opioid and non-barbiturate pain medications was $12 \%$; and $8 \%$ of enrollees were using other pharmaceuticals such as anti-hypertensives or antidepressants. Overall, the participants were generally healthy adults with chronic musculoskeletal discomfort.

At the baseline, none of the plasma biomarkers, including hsCRP, was statistically significantly correlated with the musculoskeletal discomfort assessment. Hsu et al. (2019) also reported that the level of serum hsCRP was not statistically different for chronic kidney disease patients with or without musculoskeletal pain $^{18}$. Demographic information is summarized in Table 1.

Analysis of compliance and adverse events

The level of compliance was assessed by counting the remaining pills in the returned bottles. The following three numbers indicate: $>75 \%$ compliance, $<75 \%$ compliance, and missing bottles: Verum group: $41,5,4$; Placebo group: $35,8,7$. The number of compliant subjects for both bottle groups ( $>75 \%)$ was 32 . New symptoms reported did not need urgent-care and the symptoms were resolved within three days. The adverse events reported for verum were: gasses(1), loose stool(1), GI symptoms(1), digestive issues(1), drowsiness(1), constipation(1); and for placebo: burping(1), loose stool(1), gastritis(1), low back tweak(1), drowsiness(1), and headache(1).

Crossover analysis

For the placebo effect, the baseline value was subtracted from the followup_1 value for Group A (placebo verum sequence) and the followup_1 value was subtracted from the followup_2 value for Group B (verum - 
placebo sequence). For the verum effect, the followup_1 value was subtracted from followup_2 value for Group A and the baseline value was subtracted from followup_1 value for Group B. The aggregate average of the difference within each subject $(n=50)$ were then compared between the placebo and verum by multiple t-tests. One outlier was identified by the Grubb's ESD test and excluded in series from the aggregate calculations. Using the Holm-Sidak multiple-comparison adjustment, none of the factors was statistically significantly different between the verum and placebo. (Fig. 2) The hsCRP was analyzed by LabCorp, Inc., and cytokines were analyzed by Eve Technologies Corporation, Ontario, Canada.

Fatty acid analysis

The placebo capsule, verum capsule and plasma were analyzed for 13 free fatty acids (Lipid Analytical Laboratories, Ontario, Canada). The frozen plasma specimens of seven subjects who were higher than $90 \%$ compliant by pill count were selected for fatty acid analysis to investigate whether or not an elevated quantity of n7FA was detectable when verum was dosed compared to placebo. Bradbury et al. (2011) suggested that the $16: 1$ fatty acid detected would be $16: 1 \mathrm{n} 7$ (with no 16:1n9); and for human serum/plasma lipids, the $16: 1 \mathrm{n} 9$ would be undetectable or only trace amounts detected ${ }^{19}$.

Because de novo synthesis of n7FA involves stearoyl-CoA desaturase 1 (SCD1) acting on palmitate (16:0) to produce $16: 1 \mathrm{nFA}^{20}$, the desaturation ratio $(16: 1 \mathrm{n} 7 / 16: 0)$ could be influenced by dietary intake of n7FA. However, the desaturation ratio or other free fatty acids concentrations alone did not distinguish plasma from baseline, verum or placebo. For instance, we expected an elevation of medium chain fatty acids in plasma of placebo-dosed participants; however, fluctuation in the values was negligible. We were unable to find a particular pattern in the fatty acids profile by dosing. The time of the last dosage was not documented. Because the visit appointment was usually in the morning, the last dosage was likely the night before or 10-12 hours prior to the blood draw. Two additional findings were: 1) contrary to the certificate of analysis provided (227 mg/capsule 16:1n7FA), our third-party independent analysis found the quantity of 16:1n7FA to be $344 \mathrm{mg}$ per capsule, and 2) the verum also contained $199 \mathrm{mg}$ per capsule of palmitate. Therefore, the dosage used in our verum would be $688 \mathrm{mg}$ of 16:1n7FA and $398 \mathrm{mg}$ of palmitate per day.

\section{Post-hoc analyses}

For the first post-hoc analysis, Fig. 3 indicates the baseline, followup_1, and followup_2 measurements of Group $A(n=26)$ and $B(n=24)$ for three objective and three subjective measurements. Three biomarkers demonstrated no pre-post significance for verum and placebo, whereas subjective measurement showed statistically significant reductions in Fatigue and Pain Interference measurements between the baseline and followup_1 for the placebo only; however, this reduction did not continue to the third measurement (followup_2).

Effect size considerations for future cross-over studies

The second analysis focused on the estimation of effect size or power analysis for available biological measurements purportedly modulated by n7FA dietary supplementation. The $p$-values in Table 2 show the 
results of unadjusted one sample t-tests using zero as the reference value, and thus indicating an upward or downward trend for the placebo or verum supplementation.

Criteria for considering viable biomarkers are: 1 ) the coefficient of variation (CV) of the placebo and verum is approximately equal ( $<20 \%$ difference), 2 ) CV is also small ( $<1000 \%)$, and 3 ) past literature articles linking the biomarker with omega-7 fatty acid levels. Because this analysis was post-hoc, no threshold value for the CV was set; however, in Table 2, three biomarkers were identified as a possibility: HDL, MG-CSF, and IL-8. The difference variability between placebo and verum for total cholesterol is also small; however, the CVs are large and the directional change for the mean of placebo and verum are the same. Mozaffarian, et al. (2010) reported that higher serum palmitoleate concentration was independently associated with higher HDL in 3,630 US men and women in the Cardiovascular Health Study. ${ }^{21}$ Rodriguez-Carrio et al. (2016) reported lower levels of palmitoleate in rheumatoid arthritis (RA) patients and raised levels of proinflammatory cytokines, including GM-CSF, compared to healthy controls. ${ }^{22}$ Finally, Chen et al. (2019) found elevated IL-8 in gestational diabetes patients whose inflammatory cytokines were modulated not by a single fatty acid but a profile of fatty acids, including palimitoleate. ${ }^{23}$

\section{Discussion}

Key Points are:

- Three weeks of a $688 \mathrm{mg} /$ day palmitoleate mixed fatty acids dietary supplement in a randomized single crossover trial did not produce a statistically detectable change compared to a MCT placebo dietary supplement.

- When each time point was examined, improved subjective PROMIS® measures of fatigue and pain interference were observed between the baseline and first follow-up assessment for the placebo only by Fisher's LSD adjustment $(p<0.03$, pre-post paired $F(2,75)=2.51$, 2-tailed $)$

- HDL may be a viable biomarker for testing the effect of palmitoleate supplementation with an estimated effect size of 0.21 .

This interventional anti-inflammatory claim of dietary n7FA supplementation to reduce serum hsCRP observed by Bernstein et al. (2014) has been widely used by CAM practitioners to promote n7FA supplements ${ }^{24-26}$. Other researchers have investigated anti-inflammatory effects by measuring cytokines in vitro such as TNFa, IL-6, IL-8 and Monocyte Chemoattractant Protein-1, and comparing them to inflammatory fatty acids such as oleic and palmitic acids ${ }^{27}$, or proposing the modulation of SCD $1^{28}$. Our study did not show a decreased trend for cytokines TNFa or IL-6.

As for the limitations of this study, we did not exactly replicate the Bernstein study (2014). A different n7FA product and a different baseline hsCRP range may account for the observed differences between the two studies; however, we also noted that in the Bernstein study, the effect estimate of -1.9 observed for n7FA versus placebo came to an effect size of $9.5^{11}$. This value is actually considerably larger than many pharmaceutical products, which seems extraordinary for a natural product. It is a well-described phenomenon that most natural products clinical trials have failed to show significant effect sizes beyond 
placebo $^{29}$. Given the fact that we were unable to see any difference between verum and placebo with n7FA supplementation, it would be of value for other research groups to repeat the Bernstein study as precisely as possible, given that these types of studies are used as non-FDA approved evidence of safety, tolerability and efficacy.

This study strongly suggests that researchers should pay attention to fatty acids that are absorbed into the body quickly. Neither the orally taken MCT fatty acids nor the n7FA appeared in the plasma at detectable levels after 12 hours. Bioavailability data on the essential fatty acid (n3FA) showed a maximum absorption peak at 7-10 hours after oral ingestion ${ }^{30}$. In the case of non-essential FAs, biofeedback mechanisms may play a role in controlling the body's FA concentration even within three weeks. A comparison study of a longterm low dose versus a short-term high dose non-essential FAs may provide additional information. For bioavailability detection, the analysis of red blood cell membranes would be advised for future studies.

This study also demonstrated a significant placebo effect in the subjective assessments. CAM clinical trials, including vitamins, minerals and other dietary supplements, are likely to have low effect sizes because the underlying purpose of using CAM is most often to assist the inherent healing process of the individual and this may be easily confounded by the placebo effect. From the results of this study, we suggest that scientifically validated biological surrogate measurements should be included in all CAM clinical trials. Placebo effects are often called nonspecific biological responses (NBR) because most scientists acknowledge the placebo effect is real but inconsistent and uncontrollable. Using a particular CAM modality may make the NBR more specific and predictable. We believe that CAM clinical trials can and should be designed to collect a large dataset that investigates the threshold of these specific and predictable placebo effects associated with specific modalities as a part of EBM in CAM.

When designing our study, pure 16:1n7FA was both cost-prohibitive and not safe for use as a control because the $L_{50}$ has not been established ${ }^{31}$. Dietary supplement companies regularly refer to their product as 'cis-palmitoleate,' perhaps because partially hydrogenated fatty acids or processed trans-isoforms have been shown to be harmful ${ }^{32}$. Traditionally, researchers have assumed that the endogenously produced or naturally occurring palmitoleate is the cis-isoform; however, trans-palmitoleate can be naturally produced from enzymatic shortening of dietary vaccenic acid in vivo ${ }^{33}$ as well as in dairy products, and these forms of trans-palmiteolate act like lipokine hormones ${ }^{34,35}$. A healthy French-Canadian population study found that high fat dairy intake was positively correlated with plasma trans-palmitoleate levels $(r=0.15 ; p=0.03)$, and total dairy consumption was positively correlated with plasma $\operatorname{CRP}(r=0.15 ; p=0.03)^{36}$. Other large crosssectional dietary studies report the association of plasma trans-palmitoleate with: 1) high intake of whole-fat dairy products ${ }^{37}$;2) inflammatory biomarkers in an elderly Swedish population ${ }^{13}$; and 3 ) other inflammatory markers such as hsCRP, RANTES, IL-1Ra, Interferon gamma, IL-10, PDGF-bb ${ }^{38}$. Therefore, verification of the cis-versus the trans-form of palmitoleate used in any future clinical trials on n7FA may prove essential.

From the viewpoint of clinicians, this study may be criticized for a lack of clinical insight because subjects were selected by voluntary participation rather than active selection by clinicians who might identify patients who would benefit from supplementing with n7FA in order to alleviate musculoskeletal discomfort. The authors acknowledge this limitation; however, volunteerism and transparency are the nature of human 
subjects research. As n7FA supplements are readily available and circulating without prescription or FDA approval, typical consumers are not clinically trained but self-dosing. Thus, this study focused on the typical consumer population rather than a clinically handpicked population.

\title{
Conclusions
}

Data presented in this article does not support one of the benchmark clinical trials published on n7FA dietary supplementation to lower serum hsCRP. Many questions remain about why we did not observe a lowering of the primary outcome measure for this study, hsCRP, and why we did not observe lowering of other inflammatory markers such as TNFa or IL-6. It may indeed be the case that in individuals with higher hsCRP levels ( $\mathrm{hsCRP}>3 \mathrm{mg} / \mathrm{L}$ ) that is due to higher $\mathrm{BMI}^{39,40}$, supplementation with cis-palmiteolate would lower their hsCRP levels, along with lowering subjectively assessed musculoskeletal pain; however, in our subanalysis, subjects with higher hsCRP levels showed no improvement with 3 weeks of supplementation with $688 \mathrm{mg}$ of palmiteolate, nor did they show an improvement in subjectively assessed musculoskeletal discomfort beyond placebo. Future studies should consider identifying individuals with higher hsCRP due to high BMI, the length of dosing with n7FA, and prior determination of what percent of the palmiteolate fatty acid dietary supplement is in the cisisoform. Our study suggests that for the general population with a range of hsCRP levels and subjectively assessed musculoskeletal discomfort, there is no benefit to n7FA supplementation.

\section{List Of Abbreviations}

\author{
ES Effect size
}

FA Fatty Acid

FDA Food and Drug Administration

FTC Federal Trade Commission

GM-CSF Granulocyte macrophage colony stimulating factor

HDL High density lipoprotein

hsCRP high-sensitivity C-reactive protein

IL- Interleukin-

LDL Low density lipoprotein

LSD Fisher's Least Significant Difference alpha level adjustment

MCT Medium chain triglycerides

PROMIS ${ }^{\circledR}$ Patient-Reported Outcomes Measurement Information System 
TG Triglycerides

TNFa Tumor Necrosis Factor alpha (pg/mL)

VLDL Very large low density lipoprotein

\section{Declarations}

Ethical approval and consent to participate:

Bastyr University, Office of Research Integrity IRB: 16-1558

Consent for publication:

signed IRB informed consent forms

Availability of Data and materials:

seven years from the date of publication at Bastyr University. De-identified data; however, a Data Use Agreement (DUA) should be signed before the transfer of data.

Competing interests:

This study was performed as a service contract and Barlean's Organic Oils, LLC (Ferndale, WA) was the sponsor. The authors' institution, Bastyr University, has received donations from the sponsor through the Development Office.

Funding:

Barlean's Organic Oils, LLC (sponsor) and Bastyr University (Institutional Support)

Authors' contributions:

PSA and MS contributed to data analysis, drafting or revising the article, gave final approval of the version to be published. MJB and MS participated in office visits and data collection. All authors agree to be accountable for all aspects of the work.

Acknowledgements:

The authors wish to thank Sarah Parsons, Kate Elliott, and Amber Coggins for study coordination.

Conflict of Interest:

This study was performed as a service contract and Barlean's Organic Oils, LLC (Ferndale, WA) was the sponsor. The authors' institution, Bastyr University, has received donations from the sponsor through the Development Office. 
Data sharing:

De-identified data are available upon the execution of data use agreement

\section{References}

1. FDA. Development \& Approval Process | Drugs. https://www.fda.gov/drugs/development-approvalprocess-drugs\#FDA. Published 2019. Accessed July 22, 2020.

2. Lewith GT. Funding for CAM. Bmj. 2007;335(7627):951. doi:10.1136/bmj.39388.393970.1F

3. Toupin April K, Gaboury I. A survey of Canadian regulated complementary and alternative medicine schools about research, evidence-based health care and interprofessional training, as well as continuing education. BMC Complement Altern Med. 2013;13. doi:10.1186/1472-6882-13-374

4. Fabbri A, Holland TJ, Bero LA. Food industry sponsorship of academic research: Investigating commercial bias in the research agenda. Public Health Nutr. 2018;21(18):3422-3430. doi:10.1017/S1368980018002100

5. Kelly MP, Heath I, Howick J, Greenhalgh T. The importance of values in evidence-based medicine. BMC Med Ethics. 2015;16(1):1-8. doi:10.1186/s12910-015-0063-3

6. Eissing L, Scherer T, Tödter K, Knippschild U, Willem GJ, Buurman WA, Pinnschmidt HO, Sander Ransen SS, Wolf AM, Bartelt A, Heeren J, Buettner C SL. De novo lipogenesis in human fat and liver is linked to ChREBP- $\beta$ and metabolic health. Nat Commun. 2013;4(1528):1-22. doi:10.1038/ncomms2537

7. RANDOM.ORG. True Random Number Service. https://www.random.org/lists/. Published 1998. Accessed June 3, 2017.

8. Esfarjani F, Khoshtinat $\mathrm{K}$, Zargaraan A, et al. Evaluating the rancidity and quality of discarded oils in fast food restaurants. Food Sci Nutr. 2019;7(7):2302-2311. doi:10.1002/fsn3.1072

9. Hung SSO, Slinger SJ. Effect of oxidized fish oil on the ascorbic acid nutrition of rainbow trout (Salmo gairdneri). Int J Vitam Nutr Res. 1980;50(4):393-400.

10. Bernstein AM, Roizen MF, Martinez L. Purified palmitoleic acid for the reduction of high-sensitivity Creactive protein and serum lipids: A double-blinded, randomized, placebo controlled study. J Clin Lipidol. 2014;8(6):612-617. doi:10.1016/j.jacl.2014.08.001

11. Faul F, Erdfelder E, Lang AG, Buchner A. G*Power 3: A flexible statistical power analysis program for the social, behavioral, and biomedical sciences. Behav Res Methods. 2007;39(2):175-191.

doi:10.3758/BF03193146

12. Macrae K, Stretton C, Lipina C, et al. Defining the role of DAG, mitochondrial function, and lipid deposition in palmitate-induced proinflammatory signaling and its counter-modulation by palmitoleate. J Lipid Res. 2013;54(9):2366-2378. doi:10.1194/jlr.M036996

13. Petersson H, Lind L, Hulthe J, Elmgren A, Cederholm T, Risérus U. Relationships between serum fatty acid composition and multiple markers of inflammation and endothelial function in an elderly population. Atherosclerosis. 2009;203(1):298-303. doi:10.1016/j.atherosclerosis.2008.06.020 
14. Pillon NJ, Arane K, Bilan PJ, Chiu TT, Klip A. Muscle cells challenged with saturated fatty acids mount an autonomous inflammatory response that activates macrophages. Cell Commun Signal. 2012;10:1-12. doi:10.1186/1478-811X-10-30

15. Harris PA, Taylor R, Thielke R, Payne J, Gonzalez N, Conde JG. Research electronic data capture (REDCap)-A metadata-driven methodology and workflow process for providing translational research informatics support. J Biomed Inform. 2009;42(2):377-381. doi:10.1016/j.jbi.2008.08.010

16. Patient-Reported Outcomes Measurement Information System. PROMIS Adult Profile Instruments. http://www.healthmeasures.net/images/PROMIS/manuals/PROMIS_Adult_Profile_Scoring_Manual.pdf. Accessed July 28, 2020.

17. Cleophas TJ, Zwinderman AH. Crossover studies with continuous variables: power analysis. Am J Ther. 2002;9(1):69-73. doi:10.1097/00045391-200201000-00011

18. Hsu HJ, Wu IW, Hsu KH, et al. The association between chronic musculoskeletal pain and clinical outcome in chronic kidney disease patients: a prospective cohort study. Ren Fail. 2019;41(1):257-266. doi:10.1080/0886022X.2019.1596817

19. Bradbury KE, Skeaff CM, Crowe FL, Green TJ, Hodson L. Serum fatty acid reference ranges: Percentiles from a New Zealand national nutrition survey. Nutrients. 2011. doi:10.3390/nu3010152

20. McCarthy MC, Cottam GL, Turner WW. Essential fatty acid deficiency in critically III surgical patients. Am J Surg. 1981;142(6):747-751. doi:10.1016/0002-9610(81)90326-3

21. Mozaffarian D, Cao H, King IB, et al. Circulating palmitoleic acid and risk of metabolic abnormalities and new-onset diabetes. Am J Clin Nutr. 2010;92(6):1350-1358. doi:10.3945/ajcn.110.003970

22. Rodríguez-Carrio J, Alperi-López M, López P, Ballina-García FJ, Suárez A. Non-esterified fatty acids profiling in rheumatoid arthritis: Associations with clinical features and Th1 response. PLoS One. 2016;11(8):1-17. doi:10.1371/journal.pone.0159573

23. Chen X, Stein TP, Steer RA, Scholl TO. Individual free fatty acids have unique associations with inflammatory biomarkers, insulin resistance and insulin secretion in healthy and gestational diabetic pregnant women. BMJ Open Diabetes Res Care. 2019;7(1):1-9. doi:10.1136/bmjdrc-2018-000632

24. Stengler M. Omega-7 can reduce your inflammation levels. https://www.besthealthnutritionals.com/blog/2015/04/02/omega-7-to-reduce-inflammation/. Published 2015. Accessed August 26, 2020.

25. Grossman M. Dr. Grossman's omega-7 chronic dry eye and anti-inflammatory formula. Natural Eye Care. https://www.naturaleyecare.com/shop/dr-grossmans-omega-7.html. Published 2003. Accessed August 26, 2020.

26. Morse N. Fighting Fat with Fat. SupplySide Product Education Program. http://expertomega7.com/wpcontent/uploads/2015/10/Fighting-Fat-with-Fat-Nancy-Morse.pdf. Published 2015. Accessed August 26, 2020.

27. de Souza CO, Valenzuela CA, Baker EJ, Miles EA, Rosa Neto JC, Calder PC. Palmitoleic Acid has Stronger Anti-Inflammatory Potential in Human Endothelial Cells Compared to Oleic and Palmitic Acids. Mol Nutr Food Res. 2018;62(20):e1800322. doi:10.1002/mnfr.201800322 
28. de Souza CO, Vannice GK, Rosa Neto JC, Calder PC. Is Palmitoleic Acid a Plausible Nonpharmacological Strategy to Prevent or Control Chronic Metabolic and Inflammatory Disorders? Mol Nutr Food Res. 2018;62(1). doi:10.1002/mnfr.201700504

29. Sorkin BC, Kuszak AJ, Bloss G, et al. Improving natural product research translation: From source to clinical trial. FASEB J. 2020. doi:10.1096/fj.201902143R

30. Davidson MH, Johnson J, Rooney MW, Kyle ML, Kling DF. A novel omega-3 free fatty acid formulation has dramatically improved bioavailability during a low-fat diet compared with omega-3-acid ethyl esters: The ECLIPSE (Epanova ${ }^{\circledR}$ compared to Lovaza ${ }^{\circledR}$ in a pharmacokinetic single-dose evaluation) study. J Clin Lipidol. 2012;6(6):573-584. doi:10.1016/j.jacl.2012.01.002

31. Morse N. A toxicity / safety assessment of dietary palmitoleic acid (POA ). Table of Contents Figures and Tables. 2016;(August):1-44.

32. Li C, Cobb LK, Vesper HW, Asma S. Global surveillance of trans-fatty acids. Prev Chronic Dis. 2019;16(10):1-5. doi:10.5888/pcd16.190121

33. Jaudszus A, Kramer R, Pfeuffer M, Roth A, Jahreis G, Kuhnt K. Trans Palmitoleic acid arises endogenously from dietary vaccenic acid. Am J Clin Nutr. 2014;99(3):431-435. doi:10.3945/ajcn.113.076117

34. Frigolet ME, Gutiérrez-Aguilar R. The Role of the Novel Lipokine Palmitoleic Acid in Health and Disease. Am Soc Nutr. 2017;5(20):173-181. doi:10.3945/an.115.011130.FIGURE

35. Guillocheau E, Legrand P, Rioux V. Trans-palmitoleic acid (trans-9-C16:1, or trans-C16:1 n-7): Nutritional impacts, metabolism, origin, compositional data, analytical methods and chemical synthesis. A review. Biochimie. 2020;169:144-160. doi:10.1016/j.biochi.2019.12.004

36. Da Silva MS, Julien P, Couture P, Lemieux S, Vohl MC, Rudkowska I. Associations between dairy intake and metabolic risk parameters in a healthy french-Canadian population. Appl Physiol Nutr Metab. 2014;39(12):1323-1331. doi:10.1139/apnm-2014-0154

37. Mozaffarian D, De Oliveira Otto MC, Lemaitre RN, et al. Trans-Palmitoleic acid, other dairy fat biomarkers, and incident diabetes: The multi-ethnic study of atherosclerosis (MESA). Am J Clin Nutr. 2013;97(4):854-861. doi:10.3945/ajcn.112.045468

38. Perreault M, Roke K, Badawi A, et al. Plasma levels of 14:0, 16:0, 16:1n-7, and 20:3n-6 are positively associated, but 18:0 and 18:2n-6 are inversely associated with markers of inflammation in young healthy adults. Lipids. 2014;49(3):255-263. doi:10.1007/s11745-013-3874-3

39. Aronson D, Sella R, Sheikh-Ahmad M, et al. The association between cardiorespiratory fitness and Creactive protein in subjects with the metabolic syndrome. J Am Coll Cardiol. 2004;44(10):2003-2007. doi:10.1016/j.jacc.2004.08.030

40. Visser M, Mcquillan GM, Wener MH, Harris TB. Elevated C-Reactive Protein Levels. 2020.

\section{Tables}

Table 1: * indicates the significance at the alpha level $p \leq 0.05$. In general, subjects in Group B appeared to display better lipid profiles; however, this should not be a critical randomization error for a cross-over design. 
The type of diet and exercise frequencies reported showed that the participants were fairly health conscious. For instance, the ketogenic diet was a trendy diet in 2017 , and $14 \%$ were vegetarian or vegetable-based diets. More than $50 \%$ of participants exercise at least every other day. Multiple t-tests were adjusted by the HolmsSidak method.

\begin{tabular}{|c|c|c|c|}
\hline Table 1 & Group A ( $n=26)$ & Group B $(n=24)$ & Multiple t-test \\
\hline Age & $55.5 \pm 13.2$ & $56.3 \pm 14.4$ & 0.84 \\
\hline Female & 20 (6 males) & 21 (3 males) & $\mathrm{n} / \mathrm{a}$ \\
\hline BMI $\left(\mathrm{kg} / \mathrm{m}^{2}\right)$ & $30.4 \pm 7.6$ & $28.2 \pm 7.0$ & 0.29 \\
\hline Cannabis use & 5 & 4 & $\mathrm{n} / \mathrm{a}$ \\
\hline \multicolumn{4}{|c|}{ Type of Diet (n) } \\
\hline $\begin{array}{l}\text { Type of diet } \\
\text { reported }\end{array}$ & $\begin{array}{c}\text { Ketogenic/Low carb (4) } \\
\text { Vegetable-based (2) } \\
\text { Gluten free (3) } \\
\text { Low fat (1) } \\
\text { Other healthy (5) } \\
\text { Std American (11) }\end{array}$ & $\begin{array}{l}\text { Ketogenic/low carb (5) } \\
\text { Vegetable-based (5) } \\
\text { Gluten free (1) } \\
\text { Low fat (2) } \\
\text { Other healthy (3) } \\
\text { Std American (8) }\end{array}$ & $\mathrm{n} / \mathrm{a}$ \\
\hline \multicolumn{4}{|c|}{ Exercise Frequencies } \\
\hline Never & 2 & 2 & n/a \\
\hline $1-2 \times$ per month & 1 & 2 & $\mathrm{n} / \mathrm{a}$ \\
\hline Once a week & 4 & 5 & $\mathrm{n} / \mathrm{a}$ \\
\hline $2-3 \times$ per week & 7 & 5 & n/a \\
\hline $4-5 \times$ per week & 10 & 7 & $\mathrm{n} / \mathrm{a}$ \\
\hline$>5 \times$ per week & 2 & 3 & n/a \\
\hline \multicolumn{4}{|c|}{ Biological Measurements (mean \pm std dev) } \\
\hline hsCRP (mg/L) & $3.7 \pm 3.8$ & $2.2 \pm 1.9$ & 0.10 \\
\hline Total Cholesterol & $222.0 \pm 37.9$ & $208.3 \pm 45.2$ & 0.25 \\
\hline $\mathrm{HDL}(\mathrm{mg} / \mathrm{dL})$ & $54.7 \pm 15.0$ & $65.5 \pm 20.4$ & $0.04^{*}$ \\
\hline LDL (mg/dL) & $130.3 \pm 35.3$ & $115.1 \pm 43.5$ & 0.18 \\
\hline VLDL (mg/dL) & $37.0 \pm 17.2$ & $27.8 \pm 16.2$ & 0.06 \\
\hline TG (mg/dL) & $184.8 \pm 87.7$ & $139.3 \pm 81.5$ & $0.01^{*}$ \\
\hline TNF (pg/mL) & $16.3 \pm 11.0$ & $17.8 \pm 15.2$ & 0.68 \\
\hline IL-6 (pg/mL) & $1.3 \pm 1.7$ & $0.9 \pm 1.0$ & 0.30 \\
\hline
\end{tabular}

Table 2: The major constituents of placebo capsules were capryliate (8:0), capriate (10:0), and laurate (12:0); whereas the major constituents of verum capsules were palmitate (16:0), palmitoleate (16:1), Eicosapentaenoic acid (EPA, 20:5n3), and Docosahexaenoic acid (DHA, 22:6n3). Statistical analysis (ANOVA) demonstrated that none of the fatty acids listed differed in the plasma at the baseline, nor during placebo and verum dosing. In other words, although subjects were taking a palmitoleate-rich capsule during the verum dosing, the plasma fatty acid profile did not show a statistical difference. $(n=7$ who were $>95 \%$ compliant by pill count). 


\begin{tabular}{|l|c|c|c|c|c|}
\hline & $\begin{array}{c}\text { Placebo capsule } \\
(\mathrm{mg} / \mathrm{g})\end{array}$ & $\begin{array}{c}\text { Verum capsule } \\
(\mathrm{mg} / \mathrm{g})\end{array}$ & $\begin{array}{c}\text { Baseline plasma } \\
(\mu \mathrm{g} / \mathrm{mL})\end{array}$ & $\begin{array}{c}\text { Placebo plasma } \\
(\mathbf{\mu g} / \mathrm{mL})\end{array}$ & $\begin{array}{c}\text { Verum plasma } \\
\text { (ug/mL) }\end{array}$ \\
\hline $\mathrm{C} 8: 0$ & $526.58 \pm 0.12$ & $9.74 \pm 1.13$ & $0.04 \pm 0.10$ & $0.09 \pm 0.24$ & $0.00 \pm 0.00$ \\
\hline $\mathrm{C} 10: 0$ & $359.20 \pm 0.76$ & $6.15 \pm 0.78$ & $1.05 \pm 0.73$ & $1.19 \pm 1.28$ & $1.02 \pm 0.92$ \\
\hline $\mathrm{C} 12: 0$ & $0.44 \pm 0.00$ & $0.04 \pm 0.01$ & $0.86 \pm 1.89$ & $0.61 \pm 1.25$ & $0.12 \pm 0.08$ \\
\hline $\mathrm{C} 16: 0$ & $0.23 \pm 0.03$ & $261.65 \pm 0.91$ & $1065.96 \pm 292.45$ & $995.13 \pm 210.52$ & $936.40 \pm 308.84$ \\
\hline $\mathrm{C} 16: 1 \mathrm{n} 7$ & $2.14 \pm 0.10$ & $452.16 \pm 1.72$ & $84.68 \pm 41.96$ & $71.40 \pm 37.19$ & $78.29 \pm 46.55$ \\
\hline $\mathrm{C} 20: 5 \mathrm{n} 3$ & $0.10 \pm 0.07$ & $11.71 \pm 0.23$ & $29.81 \pm 14.85$ & $31.62 \pm 18.79$ & $27.04 \pm 10.08$ \\
\hline $\mathrm{C} 22: 6 \mathrm{n} 3$ & $0.21 \pm 0.07$ & $4.92 \pm 0.17$ & $71.73 \pm 53.74$ & $78.09 \pm 46.40$ & $61.11 \pm 23.85$ \\
\hline Total & $891.28 \pm 0.01$ & $761.92 \pm 2.34$ & $\mathrm{n} / \mathrm{a}$ & $\mathrm{n} / \mathrm{a}$ & $\mathrm{n} / \mathrm{a}$ \\
\hline
\end{tabular}

Table 3: Objective biomarker measurements in this study are listed in the left column. $\mathrm{N}=$ number of data points, $\mathrm{p}$-value $=$ unadjusted one sample t-test, $\mathrm{ES}=$ effect size for one sample t-test, $\mathrm{CV}=$ coefficient of variation, $P-V=$ the difference in $E S$ between placebo and verum. ES calculation for viable factors was determined by a: 1) smaller $C V, 2$ ) smaller $P-V$, and 3) supportive of a link between palmitoleate in published literature. Calculated ES for the palmitoleate dosed in this study for HDL was 0.21 , requiring a sample size of 147 in order to achieve a statistical power of 0.80 at alpha level 0.05 . Likewise, 184 and 614 sample sizes are needed for GM-CSF and IL-8.

\begin{tabular}{|c|c|c|c|c|c|c|c|}
\hline & $\mathrm{N}$ & p-value & Mean & Std. Deviation & ES & $\mathrm{CV}$ & $P-V$ \\
\hline hsCRP_P & 49 & 0.78 & -0.07 & 1.70 & & $2448 \%$ & \multirow{2}{*}{$59.7 \%$} \\
\hline hsCRP_V & 50 & 0.26 & 0.24 & 1.51 & & $618 \%$ & \\
\hline TG_P & 49 & 0.71 & 4.12 & 77.91 & & $1890 \%$ & \multirow{2}{*}{$46.2 \%$} \\
\hline TG_V & 50 & 0.31 & -9.86 & 68.65 & & $696 \%$ & \\
\hline TC_P & 49 & 0.49 & -2.10 & 21.19 & & $1008 \%$ & \multirow{2}{*}{$0.9 \%$} \\
\hline TC_V & 50 & 0.49 & -1.62 & 16.62 & & $1026 \%$ & \\
\hline HDL_P & 49 & 0.08 & -1.69 & 6.64 & & $392 \%$ & \multirow{2}{*}{$10.6 \%$} \\
\hline HDL_V & 50 & 0.15 & 1.38 & 6.69 & 0.21 & $485 \%$ & \\
\hline VLDL_P & 47 & 0.63 & 0.96 & 13.56 & & $1417 \%$ & \multirow{2}{*}{$55.4 \%$} \\
\hline VLDL_V & 49 & 0.09 & -2.94 & 11.95 & & $407 \%$ & \\
\hline LDL_P & 47 & 0.81 & -0.74 & 21.70 & & $2914 \%$ & \multirow{2}{*}{$22.8 \%$} \\
\hline LDL_V & 49 & 0.88 & -0.37 & 17.04 & & $4638 \%$ & \\
\hline TNFa_P & 49 & 0.67 & -0.30 & 4.85 & & $1628 \%$ & \multirow{2}{*}{$65.7 \%$} \\
\hline TNFa_V & 50 & 0.04 & 0.94 & 3.16 & & $337 \%$ & \\
\hline GM-CSF_P & 48 & 0.34 & -0.30 & 2.22 & & $729 \%$ & \multirow{2}{*}{$14.6 \%$} \\
\hline GM-CSF_V & 50 & 0.20 & 0.51 & 2.77 & 0.18 & $543 \%$ & \\
\hline MCP1_P & 49 & 0.71 & -2.18 & 41.65 & & $1907 \%$ & \multirow{2}{*}{$70.0 \%$} \\
\hline MCP1_V & 50 & 0.04 & 13.45 & 45.24 & & $336 \%$ & \\
\hline IL6_P & 49 & 0.33 & -0.21 & 1.49 & & $711 \%$ & \multirow{2}{*}{$69.6 \%$} \\
\hline IL6_V & 50 & 0.86 & 0.04 & 1.45 & & $3973 \%$ & \\
\hline IL-8_P & 49 & 0.33 & -0.35 & 2.49 & & $715 \%$ & \multirow{2}{*}{$16.2 \%$} \\
\hline IIL-8_V & 50 & 0.48 & 0.20 & 1.99 & 0.10 & $992 \%$ & \\
\hline IL-10_P & 49 & 0.23 & -0.76 & 4.36 & & $576 \%$ & \multirow{2}{*}{$66.6 \%$} \\
\hline IL-10_V & 49 & 0.81 & 0.07 & 2.15 & & $2871 \%$ & \\
\hline
\end{tabular}

\section{Figures}




\section{Trial Recruitment Flow Chart}

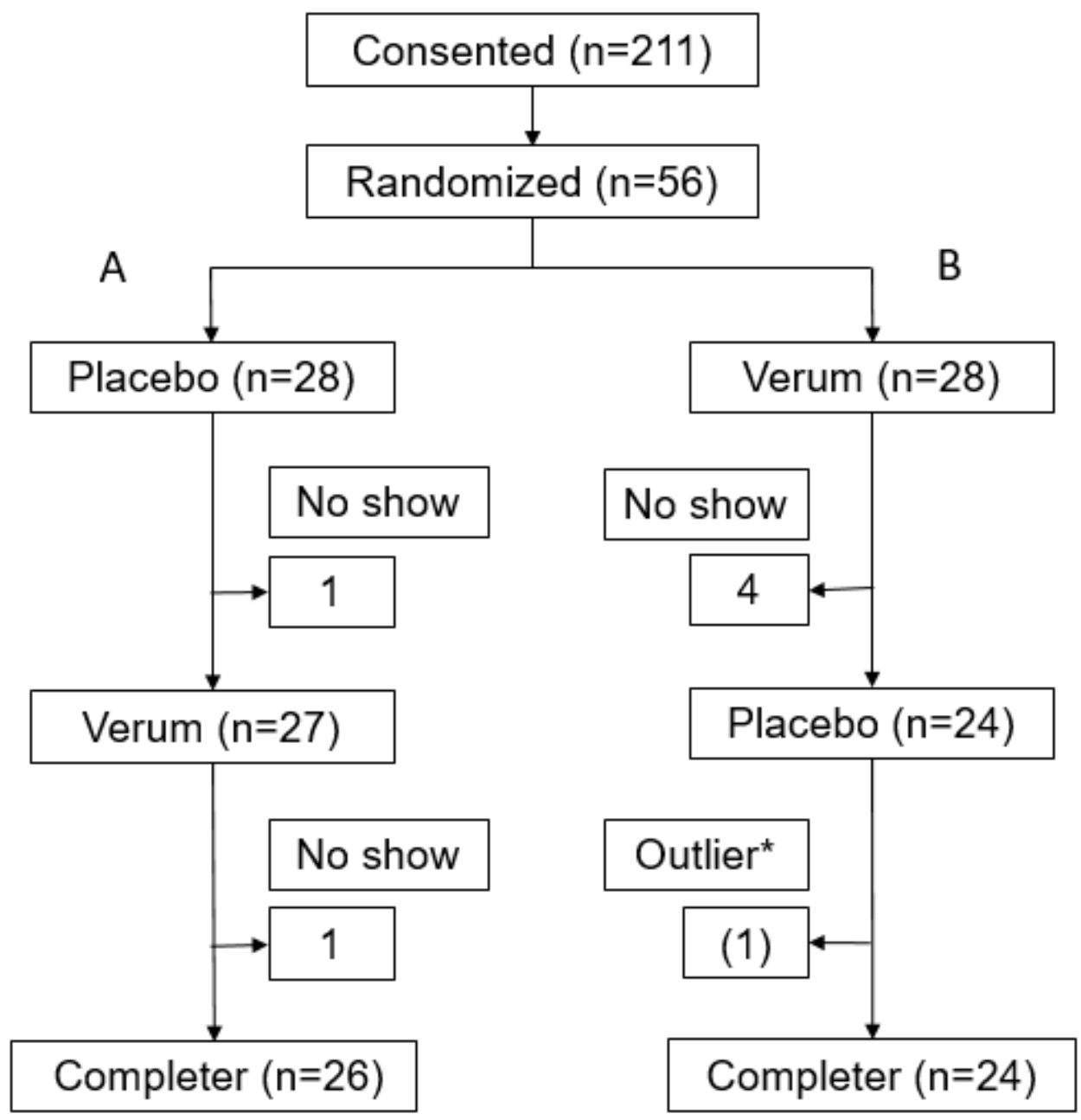

Figure 1

With the single crossover design, subjects who participated in both verum and placebo measurements were included in the analysis per protocol. * One hsCRP data point was detected as an outlier by the Grubb's HSD at the alpha $=0.05$. This subject reported a flu episode on the date when the outlier was detected. This subject was not excluded; however, for aggregate statistics, the data points (follow-up 2) of this subject were excluded in series. 


\section{Trial Recruitment Flow Chart}

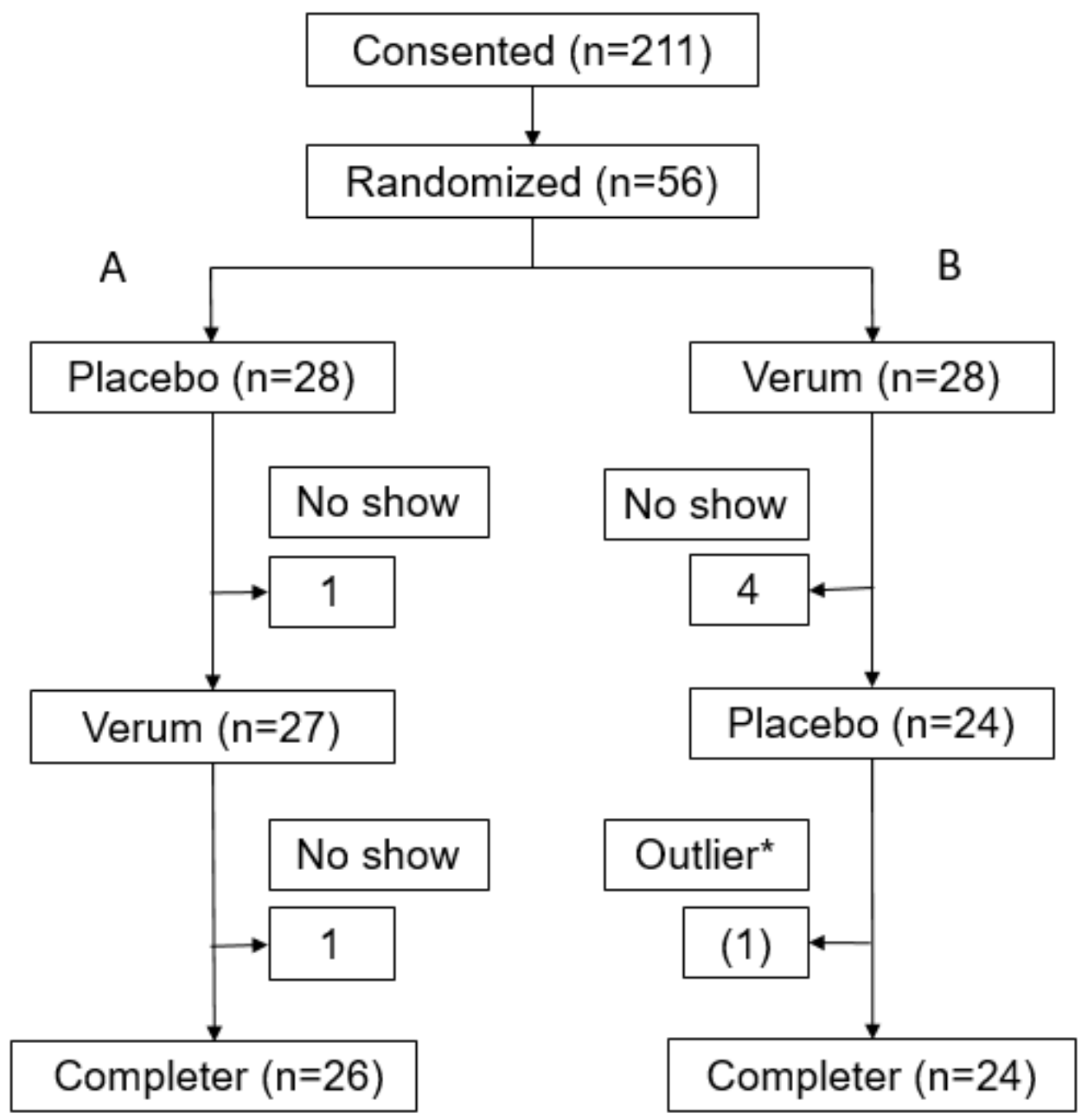

Figure 1

With the single crossover design, subjects who participated in both verum and placebo measurements were included in the analysis per protocol. * One hsCRP data point was detected as an outlier by the Grubb's HSD at the alpha $=0.05$. This subject reported a flu episode on the date when the outlier was detected. This subject was not excluded; however, for aggregate statistics, the data points (follow-up 2) of this subject were excluded in series. 


\section{Trial Recruitment Flow Chart}

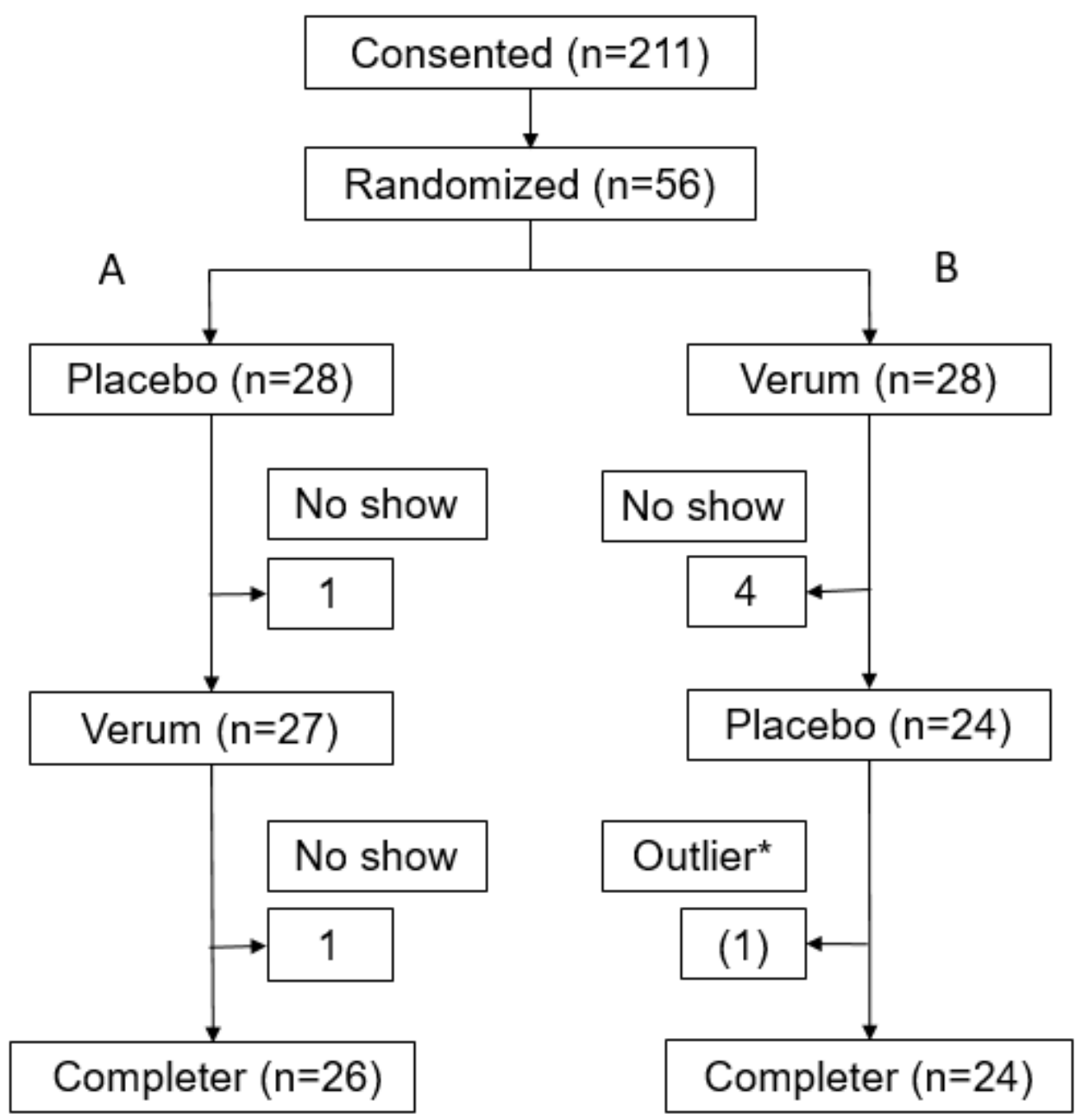

Figure 1

With the single crossover design, subjects who participated in both verum and placebo measurements were included in the analysis per protocol. * One hsCRP data point was detected as an outlier by the Grubb's HSD at the alpha $=0.05$. This subject reported a flu episode on the date when the outlier was detected. This subject was not excluded; however, for aggregate statistics, the data points (follow-up 2) of this subject were excluded in series. 


\section{Crossover aggregates of placebo versus verum data contributed by each individual, $n=50$}

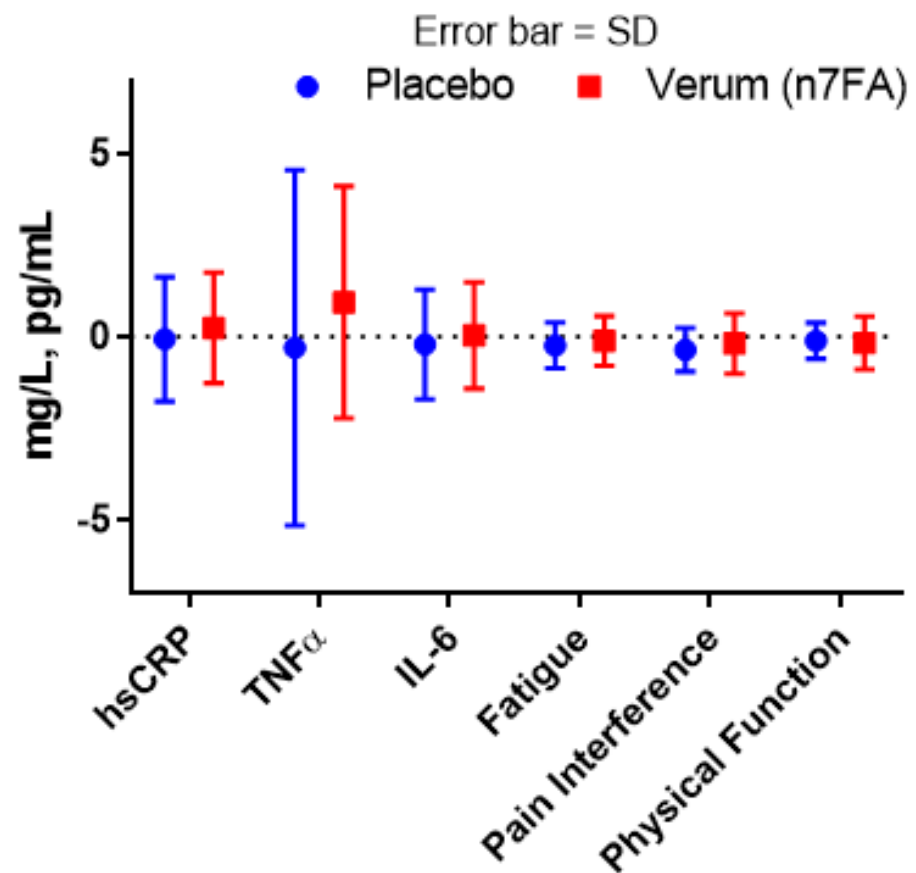

\begin{tabular}{|c|c|c|c|c|c|}
\hline Figure 2 & $\mathbf{M} \pm \mathbf{S D}$ & $\mathbf{n}$ & Difference \pm SEM & Df & $\begin{array}{l}\text { Holm-Sidak method } \\
\text { adjusted p-value }\end{array}$ \\
\hline hsCRP_P & $-0.07 \pm 1.70$ & 49 & \multirow{2}{*}{$-0.31 \pm 0.32$} & \multirow{2}{*}{97} & \multirow{2}{*}{0.80} \\
\hline hsCRP_V & $0.24 \pm 1.51$ & 50 & & & \\
\hline TNFa_P & $-0.30 \pm 4.85$ & 49 & \multirow{2}{*}{$-1.24 \pm 0.82$} & \multirow{2}{*}{97} & \multirow{2}{*}{0.58} \\
\hline TNFa_V & $0.94 \pm 3.16$ & 50 & & & \\
\hline IL6_P & $-0.21 \pm 1.50$ & 49 & \multirow{2}{*}{$-0.25 \pm 0.30$} & \multirow{2}{*}{97} & \multirow{2}{*}{0.80} \\
\hline IL6_V & $0.04 \pm 1.45$ & 50 & & & \\
\hline Fatigue_P & $-0.23 \pm 0.62$ & 49 & \multirow{2}{*}{$-0.13 \pm 0.13$} & \multirow{2}{*}{97} & \multirow{2}{*}{0.80} \\
\hline Fatigue_V & $-0.11 \pm 0.68$ & 50 & & & \\
\hline PainInt_P & $-0.35 \pm 0.59$ & 49 & \multirow{2}{*}{$-0.17 \pm 0.14$} & \multirow{2}{*}{97} & \multirow{2}{*}{0.76} \\
\hline PainInt_V & $-0.18 \pm 0.82$ & 50 & & & \\
\hline PhyFunc_P & $-0.11 \pm 0.49$ & 49 & \multirow{2}{*}{$0.06 \pm 0.12$} & \multirow{2}{*}{97} & \multirow{2}{*}{0.80} \\
\hline PhyFunc_V & $-0.17 \pm 0.73$ & 50 & & & \\
\hline
\end{tabular}

\section{Figure 2}

Fifty data points were provided from 26 subjects who were assigned to taking the placebo-verum sequence and 24 subjects who were assigned to the verum-placebo sequence. A mixed t-test or split-plot analysis showed no significant difference between the verum and placebo measurements in both biomarkers and subjective assessments. One data point in placebo measurement was found an outlier by Grubb's ESD test at alpha level of 0.05 . The multiple t-tests were adjusted by the Holm-Sidak adjustment. Painlnt = pain interference. PhyFunc $=$ physical functioning. $\mathrm{P}=$ placebo. $\mathrm{V}=$ verum . 


\section{Crossover aggregates of placebo versus verum data contributed by each individual, $n=50$}

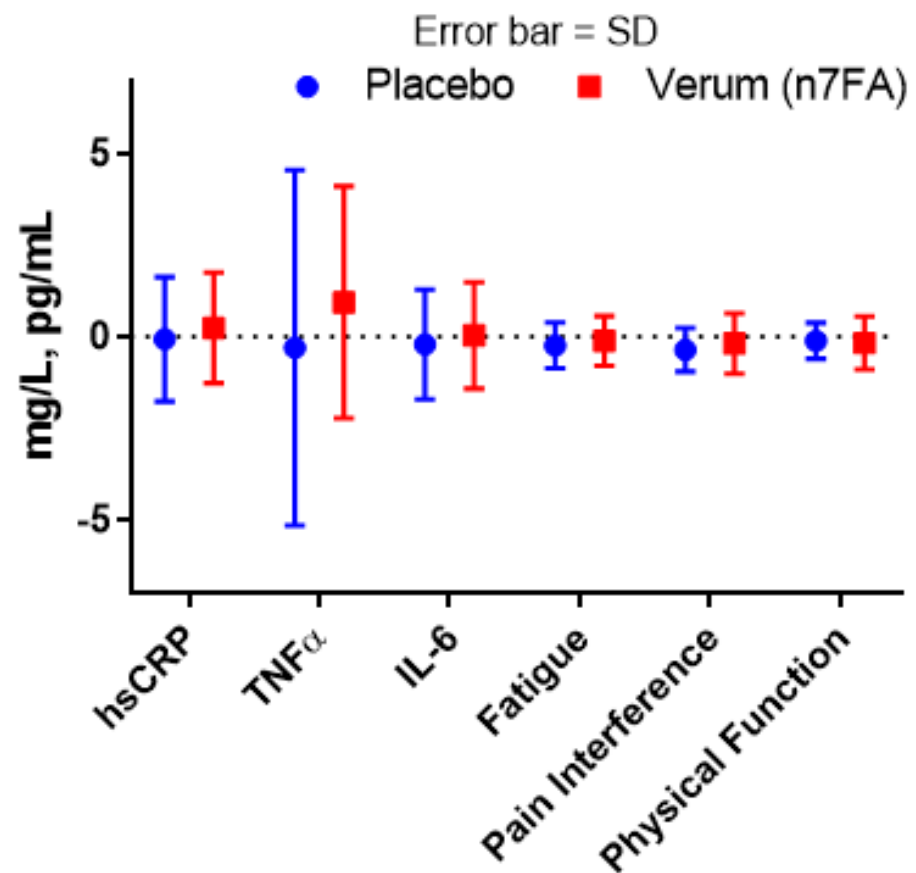

\begin{tabular}{|c|c|c|c|c|c|}
\hline Figure 2 & $\mathbf{M} \pm \mathbf{S D}$ & $\mathbf{n}$ & Difference \pm SEM & Df & $\begin{array}{l}\text { Holm-Sidak method } \\
\text { adjusted p-value }\end{array}$ \\
\hline hsCRP_P & $-0.07 \pm 1.70$ & 49 & \multirow{2}{*}{$-0.31 \pm 0.32$} & \multirow{2}{*}{97} & \multirow{2}{*}{0.80} \\
\hline hsCRP_V & $0.24 \pm 1.51$ & 50 & & & \\
\hline TNFa_P & $-0.30 \pm 4.85$ & 49 & \multirow{2}{*}{$-1.24 \pm 0.82$} & \multirow{2}{*}{97} & \multirow{2}{*}{0.58} \\
\hline TNFa_V & $0.94 \pm 3.16$ & 50 & & & \\
\hline IL6_P & $-0.21 \pm 1.50$ & 49 & \multirow{2}{*}{$-0.25 \pm 0.30$} & \multirow{2}{*}{97} & \multirow{2}{*}{0.80} \\
\hline IL6_V & $0.04 \pm 1.45$ & 50 & & & \\
\hline Fatigue_P & $-0.23 \pm 0.62$ & 49 & \multirow{2}{*}{$-0.13 \pm 0.13$} & \multirow{2}{*}{97} & \multirow{2}{*}{0.80} \\
\hline Fatigue_V & $-0.11 \pm 0.68$ & 50 & & & \\
\hline PainInt_P & $-0.35 \pm 0.59$ & 49 & \multirow{2}{*}{$-0.17 \pm 0.14$} & \multirow{2}{*}{97} & \multirow{2}{*}{0.76} \\
\hline PainInt_V & $-0.18 \pm 0.82$ & 50 & & & \\
\hline PhyFunc_P & $-0.11 \pm 0.49$ & 49 & \multirow{2}{*}{$0.06 \pm 0.12$} & \multirow{2}{*}{97} & \multirow{2}{*}{0.80} \\
\hline PhyFunc_V & $-0.17 \pm 0.73$ & 50 & & & \\
\hline
\end{tabular}

\section{Figure 2}

Fifty data points were provided from 26 subjects who were assigned to taking the placebo-verum sequence and 24 subjects who were assigned to the verum-placebo sequence. A mixed t-test or split-plot analysis showed no significant difference between the verum and placebo measurements in both biomarkers and subjective assessments. One data point in placebo measurement was found an outlier by Grubb's ESD test at alpha level of 0.05 . The multiple t-tests were adjusted by the Holm-Sidak adjustment. Painlnt = pain interference. PhyFunc $=$ physical functioning. $\mathrm{P}=$ placebo. $\mathrm{V}=$ verum . 


\section{Crossover aggregates of placebo versus verum data contributed by each individual, $n=50$}

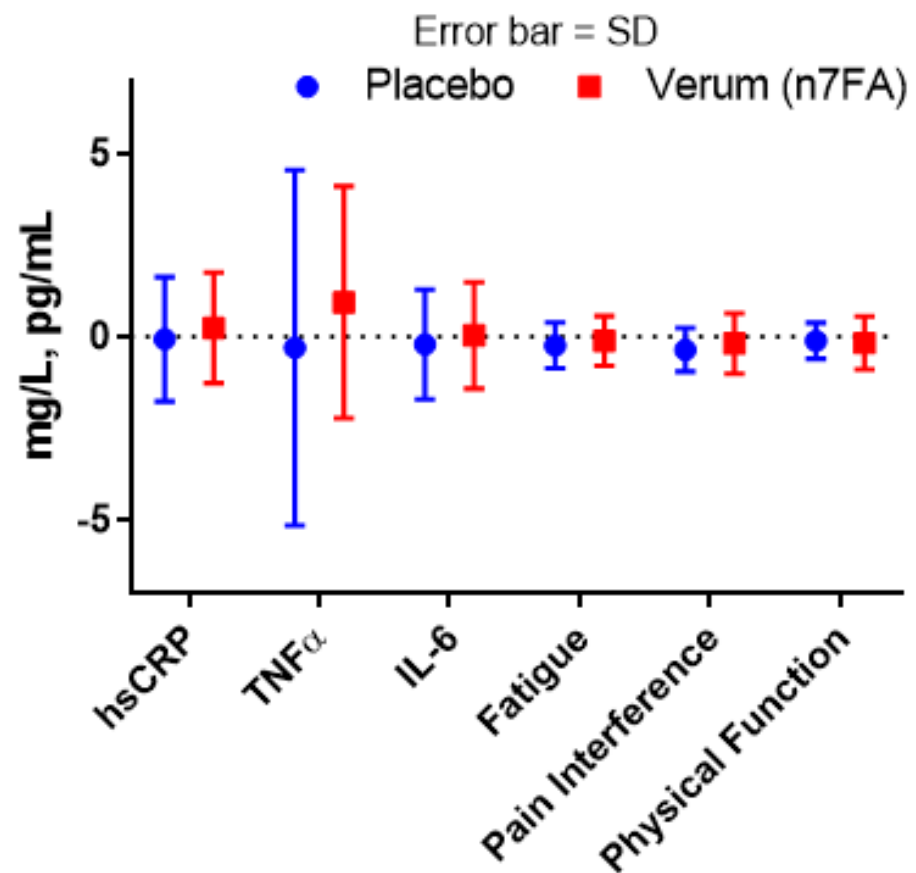

\begin{tabular}{|c|c|c|c|c|c|}
\hline Figure 2 & $\mathbf{M} \pm \mathbf{S D}$ & $\mathbf{n}$ & Difference \pm SEM & Df & $\begin{array}{l}\text { Holm-Sidak method } \\
\text { adjusted p-value }\end{array}$ \\
\hline hsCRP_P & $-0.07 \pm 1.70$ & 49 & \multirow{2}{*}{$-0.31 \pm 0.32$} & \multirow{2}{*}{97} & \multirow{2}{*}{0.80} \\
\hline hsCRP_V & $0.24 \pm 1.51$ & 50 & & & \\
\hline TNFa_P & $-0.30 \pm 4.85$ & 49 & \multirow{2}{*}{$-1.24 \pm 0.82$} & \multirow{2}{*}{97} & \multirow{2}{*}{0.58} \\
\hline TNFa_V & $0.94 \pm 3.16$ & 50 & & & \\
\hline IL6_P & $-0.21 \pm 1.50$ & 49 & \multirow{2}{*}{$-0.25 \pm 0.30$} & \multirow{2}{*}{97} & \multirow{2}{*}{0.80} \\
\hline IL6_V & $0.04 \pm 1.45$ & 50 & & & \\
\hline Fatigue_P & $-0.23 \pm 0.62$ & 49 & \multirow{2}{*}{$-0.13 \pm 0.13$} & \multirow{2}{*}{97} & \multirow{2}{*}{0.80} \\
\hline Fatigue_V & $-0.11 \pm 0.68$ & 50 & & & \\
\hline PainInt_P & $-0.35 \pm 0.59$ & 49 & \multirow{2}{*}{$-0.17 \pm 0.14$} & \multirow{2}{*}{97} & \multirow{2}{*}{0.76} \\
\hline PainInt_V & $-0.18 \pm 0.82$ & 50 & & & \\
\hline PhyFunc_P & $-0.11 \pm 0.49$ & 49 & \multirow{2}{*}{$0.06 \pm 0.12$} & \multirow{2}{*}{97} & \multirow{2}{*}{0.80} \\
\hline PhyFunc_V & $-0.17 \pm 0.73$ & 50 & & & \\
\hline
\end{tabular}

\section{Figure 2}

Fifty data points were provided from 26 subjects who were assigned to taking the placebo-verum sequence and 24 subjects who were assigned to the verum-placebo sequence. A mixed t-test or split-plot analysis showed no significant difference between the verum and placebo measurements in both biomarkers and subjective assessments. One data point in placebo measurement was found an outlier by Grubb's ESD test at alpha level of 0.05 . The multiple t-tests were adjusted by the Holm-Sidak adjustment. Painlnt = pain interference. PhyFunc $=$ physical functioning. $\mathrm{P}=$ placebo $. \mathrm{V}=$ verum . 
hsCRP Biomarker over three measurements

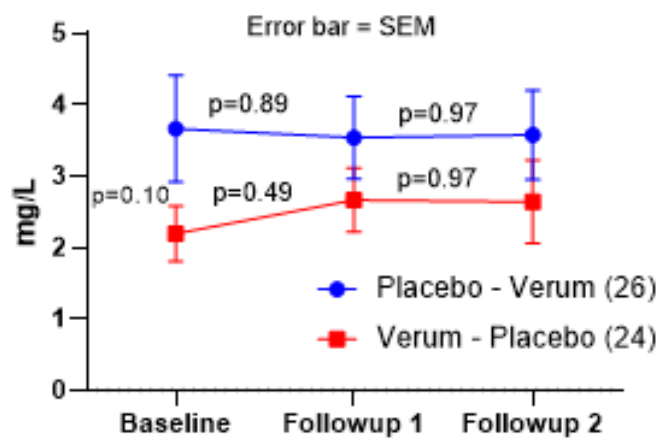

TNF $\alpha$ Biomarker over three measurements

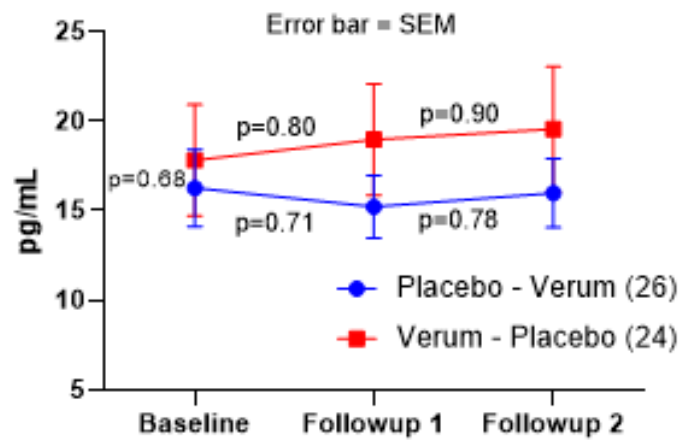

\section{IL-6 Biomarker over} three measurements

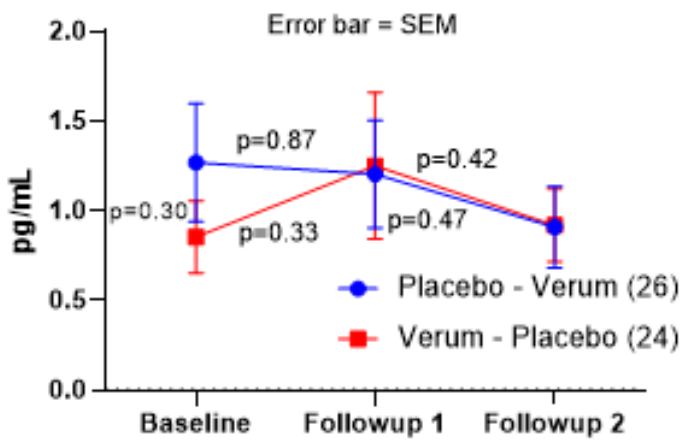

\section{Fatigue, higher the value} more fatigue

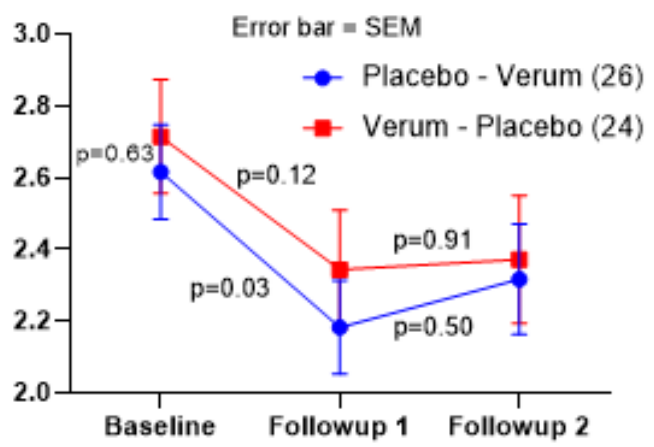

\section{Pain Interference, higher the value more interference}

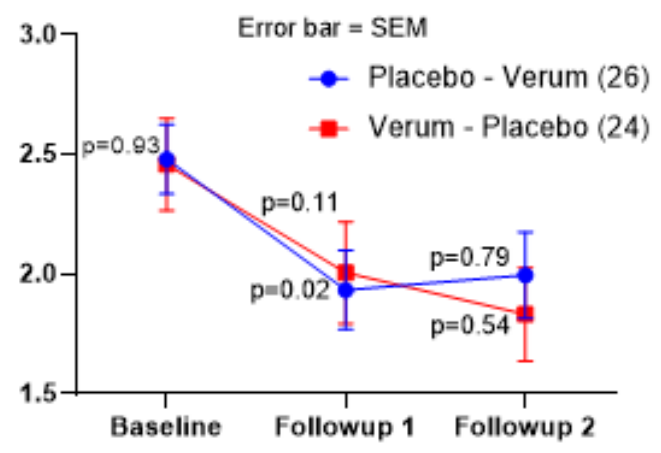

Physical Difficulties, higher the value more difficulties

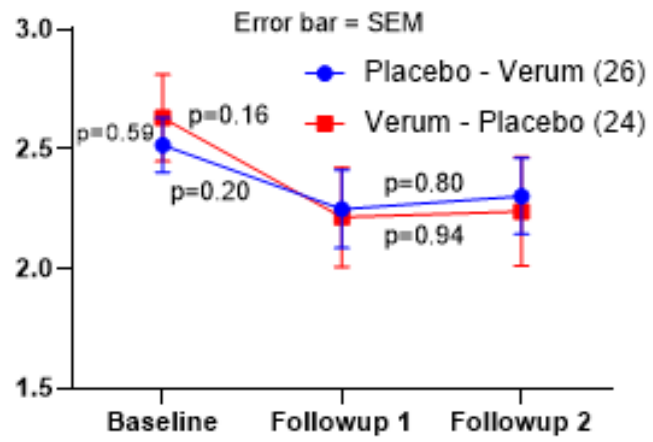

Figure 3

The graphs on the left side are the biomarkers: hsCRP, TNFa and IL-6. The graphs on the right side are the subjective assessments: fatigue, pain interference and physical functioning. The blue line indicates Group A and the red line indicates Group B. The p-values indicate pre-post paired-t tests adjusted by the Fisher's LSD. Subjective measures demonstrated a reduction in three PROMIS ${ }^{\circledR}$ measures at the time of entry into the study to the first follow up visit; however, the reduction did not continue for either the placebo or the verum 
dosing. Statistical significance was shown for the subjective assessment of fatigue and pain interference on the placebo arm only ( $p<0.03,2$-tail test, Fisher's LSD).

hsCRP Biomarker over three measurements

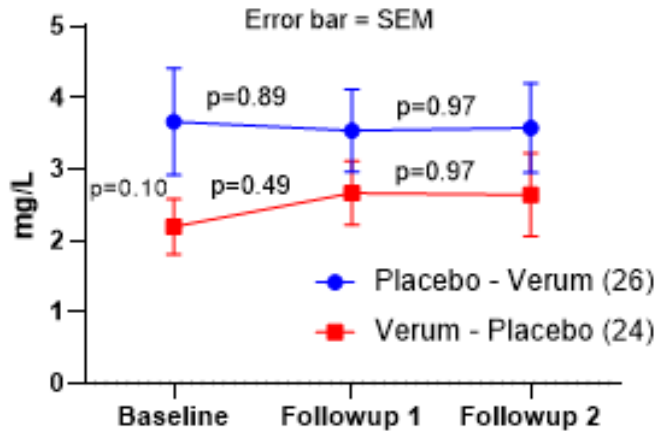

TNF $\alpha$ Biomarker over three measurements

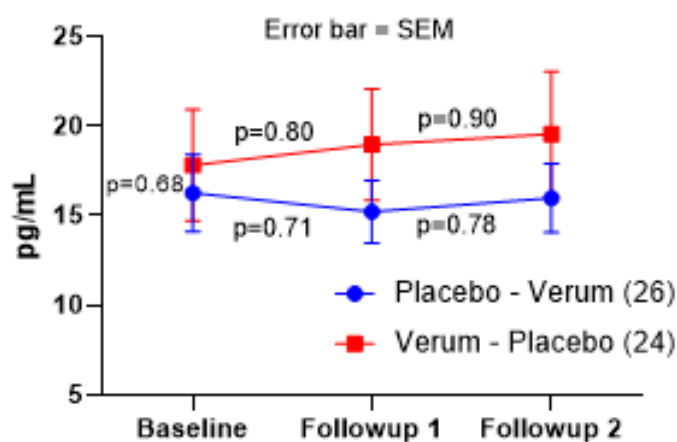

IL-6 Biomarker over three measurements

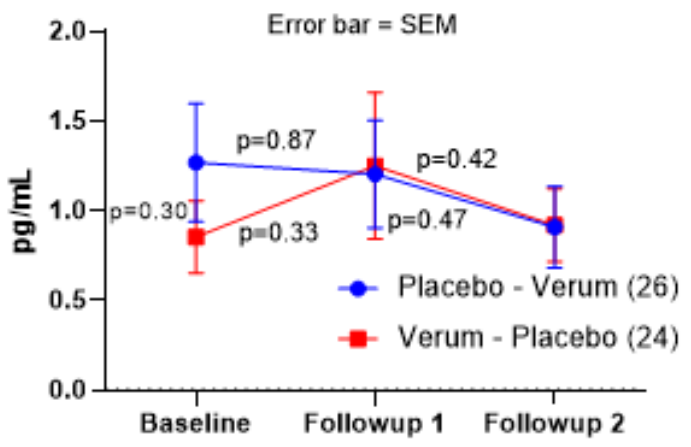

Fatigue, higher the value more fatigue

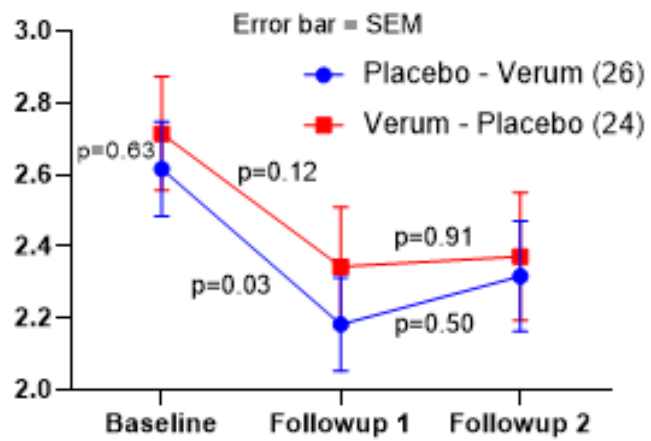

\section{Pain Interference, higher the value more interference}

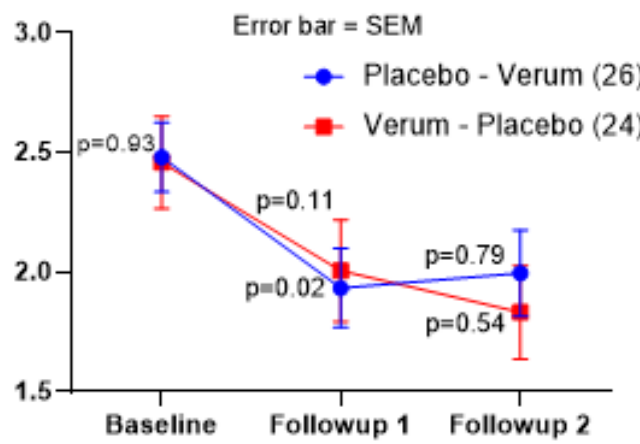

\section{Physical Difficulties, higher the value more difficulties}

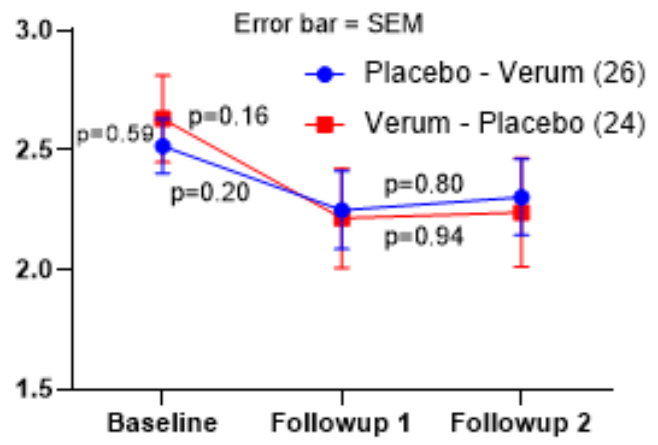

Figure 3

The graphs on the left side are the biomarkers: hsCRP, TNFa and IL-6. The graphs on the right side are the subjective assessments: fatigue, pain interference and physical functioning. The blue line indicates Group A and the red line indicates Group B. The p-values indicate pre-post paired-t tests adjusted by the Fisher's LSD. Subjective measures demonstrated a reduction in three PROMIS ${ }^{\circledR}$ measures at the time of entry into the 
study to the first follow up visit; however, the reduction did not continue for either the placebo or the verum dosing. Statistical significance was shown for the subjective assessment of fatigue and pain interference on the placebo arm only ( $p<0.03,2$-tail test, Fisher's LSD).

hsCRP Biomarker over three measurements

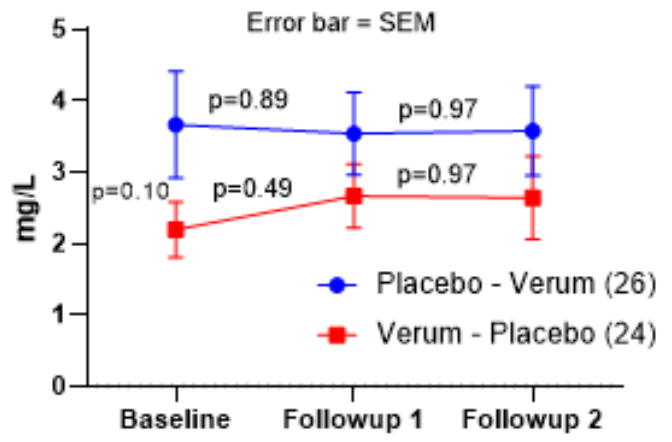

\section{TNF $\alpha$ Biomarker over} three measurements

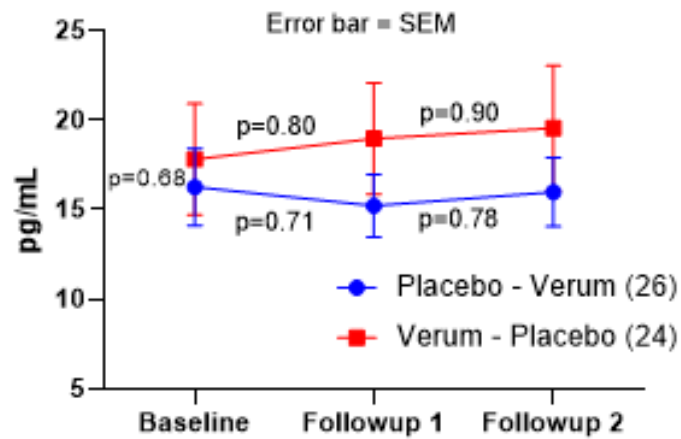

IL-6 Biomarker over three measurements

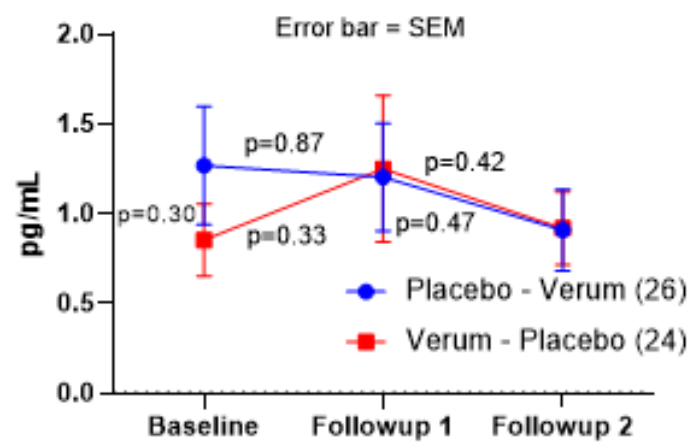

Fatigue, higher the value more fatigue

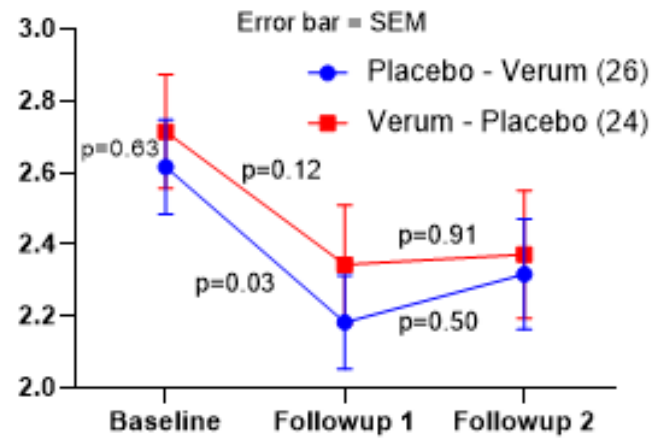

\section{Pain Interference, higher the value more interference}

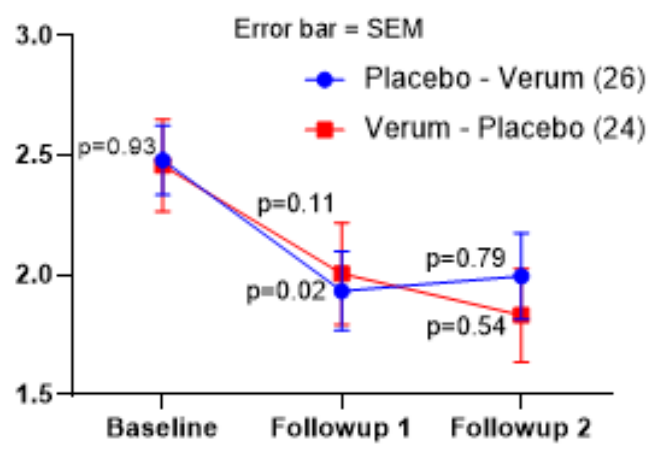

\section{Physical Difficulties, higher the value more difficulties}

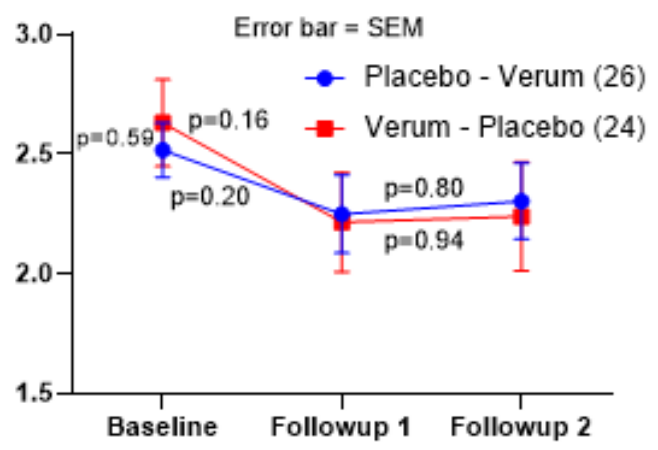

Figure 3

The graphs on the left side are the biomarkers: hsCRP, TNFa and IL-6. The graphs on the right side are the subjective assessments: fatigue, pain interference and physical functioning. The blue line indicates Group $A$ and the red line indicates Group B. The p-values indicate pre-post paired-t tests adjusted by the Fisher's LSD. 
Subjective measures demonstrated a reduction in three PROMIS ${ }^{\circledR}$ measures at the time of entry into the study to the first follow up visit; however, the reduction did not continue for either the placebo or the verum dosing. Statistical significance was shown for the subjective assessment of fatigue and pain interference on the placebo arm only ( $<<0.03$, 2-tail test, Fisher's LSD).

\section{Supplementary Files}

This is a list of supplementary files associated with this preprint. Click to download.

- CONSORTChecklist20201118.doc

- CONSORTChecklist20201118.doc

- CONSORTChecklist20201118.doc 